\title{
Performance Characteristics and Temperature Compensation Method of Fluid Property Sensor Based on Tuning-Fork Technology
}

\author{
Yuan Chen, ${ }^{1,2}$ Guifu Mei, ${ }^{2}$ Huanhuan Yang, ${ }^{2}$ Fei Yu, ${ }^{2}$ and Jun Gao ${ }^{2}$ \\ ${ }^{1}$ State Key Laboratory of Robotics and System, HIT, Harbin 150001, China \\ ${ }^{2}$ School of Mechanical, Electrical \& Information Engineering, Shandong University at Weihai, Weihai 264209, China \\ Correspondence should be addressed to Yuan Chen; cyzghysy@sdu.edu.cn
}

Received 3 July 2015; Revised 24 September 2015; Accepted 4 October 2015

Academic Editor: Liling Fu

Copyright (C) 2016 Yuan Chen et al. This is an open access article distributed under the Creative Commons Attribution License, which permits unrestricted use, distribution, and reproduction in any medium, provided the original work is properly cited.

Fluid property sensor (FPS) based on tuning-fork technology is applied to the measurement of the contaminant level of lubricant oil. The measuring principle of FPS sensor is derived and proved together with its resolution. The performance characteristics of the FPS sensor, such as sensitivity coefficient, resolution, and quality factor, are analyzed. A temperature compensation method is proposed to eliminate the temperature-dependence of the measuring parameters, and its validity is investigated by numerical simulation of sensitivity, oscillating frequency, and dielectric constant. The values of purification efficiency obtained using microwave and without microwave are compared experimentally.

\section{Introduction}

Contaminants in lubricant oil can accelerate the wear rate of machine parts. The effectiveness of nearly three-quarters of lubricant systems is reduced by contaminated lubricant oil [1]. Water, solid particulate, and gas are three main contaminants in lubricant oil. These contaminants can be removed by gravitational separation, centrifugal separation, impingement, or filters [2-4]. Gravitational separation technique is unable to separate contaminant from lubricant oil of high viscosity [5]. Centrifugal purifier can improve the separation efficiency of free water and solid particulates [6]. However, it is impossible to extract dissolved gas of low density from multiphase mixtures through centrifugal separation method. Centrifugal vacuum separation technique has been extensively applied to the removal of free water and solid particulates in various fields [7-10]. However, the removal of dissolved gas of low density from multiphase mixtures has been seldom explored. We developed a vacuum centrifugal solid-liquid-gas purifier, as shown in Figure 1 [11]. Under centrifugal force and negative pressure, free water and solid particulates can be extracted, and even dissolved gas in contaminated lubricant oil can also be removed.
The purifier is composed of the following parts: hull (1), foundation (2), disk (3), axis (4), oil tanker (5), central tube (6), inlet of contaminant (7), exit of clean oil (8), exit of bubble (9), vacuum zone (10), centrifugal purifier (11), and electromotor (12). A vacuum zone is formed when the hull spins at a great velocity. Under strong centrifugal force, free water and solid particulates are centrifuged to the interior wall of the hull. Meanwhile, the dissolved gas moves to the vacuum zone with the help of vacuum pump.

Online measurement of contaminant level in the purifier is vital to improve its separation efficiency. Weighting method, particle analysis, ferrographic technology, and semiquantitative method have been utilized to measure the contaminant level of lubricant oil. Weighting method can measure the total weight of contaminant, but it cannot determine the distribution of contaminants [12]. Particle analysis is able to measure the distribution and concentration of particle through optical microscopy and computer particle image [13]. However, this method is difficult to be embedded with online monitoring function due to its high cost and low performance in real-time analysis. Ferrographic technology can not only measure the contamination level but also calculate the morphology and concentration of particles. However, 


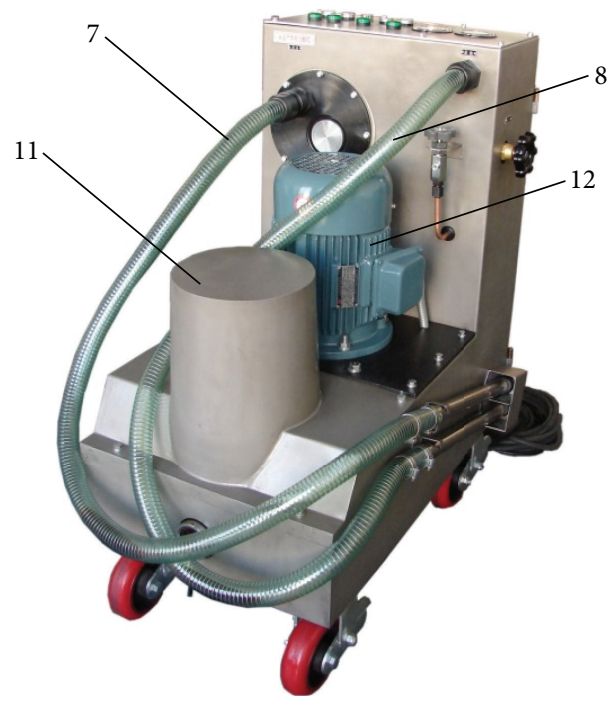

(a)

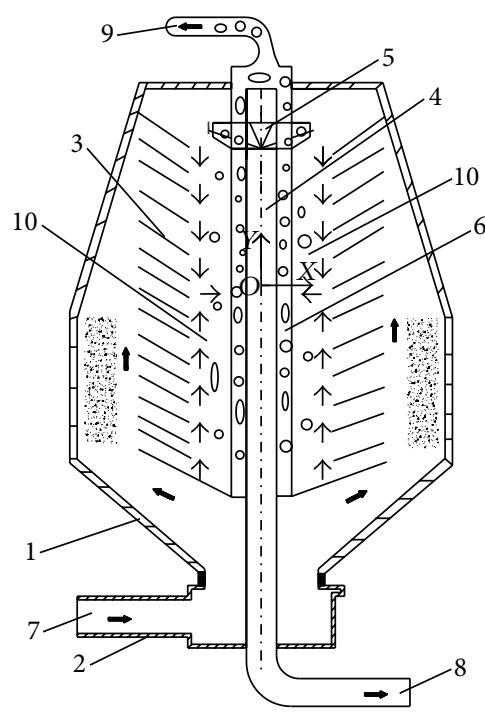

(b)

FIGURE 1: Vacuum centrifugal solid-liquid-gas purifier. (a) Configuration of purifier; (b) inner structure of purifier.

the offline monitoring and high cost of ferrographic equipment prohibit its extensive application [14]. Semiquantitative method can be roughly divided into ultrasonic, light, and sedimentation measuring methods [15]. Ultrasonic method is able to monitor the contaminants online, but it is not sensitive to particles with diameter less than $30 \mu \mathrm{m}$. The accuracy of light measuring method can be easily affected by external noise or the color of lubricant oil [16]. Although sedimentation method is not affected by the color of oil and bubble, it has the shortcomings of sophisticated operation and high cost of standard filter membrane [17].

At present, most online monitoring sensors are only capable of measuring the single physical parameters of oil medium but cannot realize the comprehensive assessment of oil quality, which shows that multiparameter measurement is the development trend for intelligent sensor technology. FPS2800B12C4 sensor (American Measurement Specialties) can simultaneously measure temperature, dielectric constant, density, and viscosity of lubricant oil. The sensor is based on quartz tuning-fork principle. However, the resonant frequency of the sensor is extremely sensitive to the change of ambient temperature. Likewise, density and viscosity are greatly dependent on temperature. Unfortunately, the purifier generates a large amount of heat during the purification of oil. Therefore, it is essential to analyze the performance characteristics of the sensor and to compensate the temperature deviation, in order to improve the accuracy and reliability of the sensor at different working temperatures. Temperature compensation method is divided into hardware solution method and soft compensation method. Some hardware auxiliary devices such as thermistor provide compensation solution of temperature $[18,19]$. However, such compensation method easily causes temperature deviation due to the distance between the main sensor and the auxiliary temperature sensor. In contrast, soft compensation method adopts mathematical model to get rid of the effect of temperature on the measured parameters. With the rapid development of intelligent sensor technology, soft compensation method has the advantages of low cost and easy implementation $[20,21]$. In order to improve the accuracy and reliability of the sensor at different working temperatures, soft temperature compensation is performed in the present paper based on the relation between the variation of dielectric constant and the temperature difference.

\section{Principle and Resolution of FPS Sensor: Theory}

2.1. Measuring Principle. Figure 2 illustrates FPS2800B12C4 sensor, which simultaneously measures density, dielectric constant, viscosity, and temperature. The ability to measure multiple parameters can improve the accuracy and reliability of the sensor. With the applied force, the resonant frequency of the sensor will vary based on the force-frequency property. When contaminants are absorbed into the lubricant oil, the resonance frequency will vary with the contaminant level. According to the physical relationship between the frequency and the measured parameters, the sensor can measure some fluid property parameters. As shown in Figure 2(b), the sensor employs the universal digital CAN J1939 protocol, so it can be easily connected to other controllers. Furthermore, it can measure the value of density ranging from 0 to $115 \mathrm{~g} / \mathrm{cm}^{3}$, dielectric constant ranging from 1100 to 6100 , viscosity ranging from 0 to $5011 \mathrm{cp}$, and temperature ranging from -40 to $150^{\circ} \mathrm{C}$.

The quartz crystal resonator inside the sensor works as a piezoelectric oscillator and generates a high-precision clock signal with quartz resonance frequency [22]. When an object is adsorbed to the surface of the quartz crystal, the oscillation frequency of the oscillator will vary with the increase of the weight of the adsorbed object. If a Newtonian liquid is in contact with one of the sensors, the change in the oscillation 


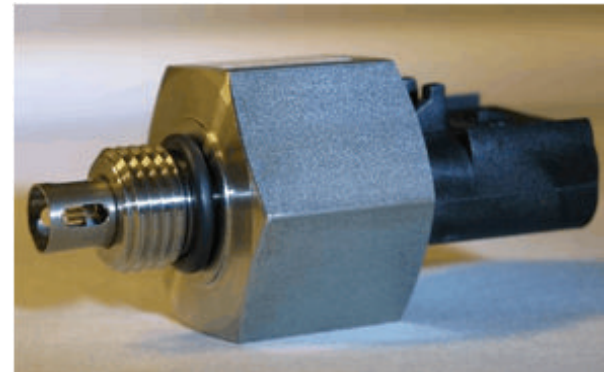

(a)

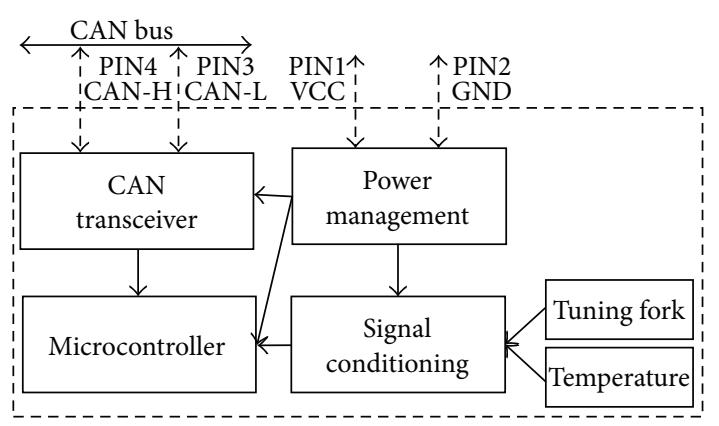

(b)

FIGURE 2: FPS2800B12C4 fluid property sensor: (a) configuration of the sensor; (b) inner structure of the sensor.

frequency is proportional to the square root of the product of the liquid density and viscosity [23]. Consider

$$
\Delta f=k \cdot \Delta \sqrt{\rho \eta},
$$

where $\Delta$ is the nominal oscillation frequency of the quartz crystal and $\rho$ and $\eta$ are the liquid density and viscosity, respectively. The oscillator sensitivity coefficient $k$ can be expressed as follows:

$$
k=-\frac{2.26 \times 10^{-6} f_{0}^{3 / 2}}{\sqrt{4 \pi}} .
$$

The sensitivity of the sensor can be improved with the increase of its nominal frequency. Solid particle, water content, and the dielectric constant of lubricant oil cause the variation of its viscosity and density. The measurement determines $\sqrt{\rho \eta}$ more accurately than it determines the density [24]. The typical nominal frequency of commercially available low-frequency quartz tuning-fork sensor is $32.768 \mathrm{KHz}$, and it is characterized by high quality factor, high precision, high stability, and low power consumption [25].

2.2. Resolution of FPS Sensor. The measurement accuracy of FPS sensor is closely related to the sensitivity coefficient $k$ in (2), the stability of oscillator frequency, and resolution of the sensor. The stability of oscillator frequency indicates the ability to maintain the oscillator frequency. Usually, the dispersion of oscillator frequency, expressed by standard deviation or variance, is used to determine the stability of oscillator frequency which varies slowly. The values of density and viscosity in purifier are independent and dispersive; this results in rapid change of oscillator frequency. The following Allan standard deviation is capable of expressing the dispersion of oscillator frequency [26]:

$$
\sigma_{y}^{2}(\tau)=\frac{1}{2 f_{0}(m-1)} \sum_{n=1}^{m-1}\left(\bar{f}_{n+1}(\tau)-\bar{f}_{n}(\tau)\right)^{2}
$$

where $f_{n}(\tau)$ is the mean oscillation frequency of the $n$th sample data during the time interval $\tau, \sigma_{y}^{2}(\tau)$ is the variation of oscillation frequency, and $m$ denotes the number of samples.
Moreover, the limit of oscillator frequency under the condition of noise is expressed by

$$
\Delta f(\tau)=\sigma_{y}(\tau) \cdot f_{0},
$$

where $f_{0}$ denotes the nominal oscillation frequency and $\sigma_{y}(\tau)$ is the Allan variance.

Then, the resolution of the FPS sensor is calculated as follows:

$$
R=\frac{\Delta f(\tau)}{k},
$$

where $R$ is the resolution of the sensor and $k$ denotes the oscillator sensitivity coefficient in (2).

The following equation presents an empirical model expressed by the relation between the smallest Allan variance and the quality factor of the resonator [27]:

$$
\sigma_{y}(\tau)_{\min }=\frac{1}{Q \times 10^{7}},
$$

where $\sigma_{y}(\tau)_{\min }$ is the smallest Allan variance, $Q$ is the quality factor of the sensor, and $\tau$ is the sampling time, generally in the range of $0.1 \mathrm{~s} \sim 10 \mathrm{~s}$.

From (6), it can be found that the smallest Allan variance is inversely proportional to the quality factor of the sensor. As fluid property sensor is operating in liquids, the damping value of resonator reduces the quality factor. Figure 3 illustrates the circuit of the quartz crystal sensor operating in a liquid. It is assumed that the sensor is an ideal quartz, namely, $R_{\mathrm{Q} 1} \approx 0$, and $L_{\mathrm{Q} 3}$ is far less than $L_{\mathrm{Q} 1}$. Then, the actual model in Figure 3(a) is reduced to the simplified model in Figure 3(b). The maximum quality factor of the resonator in the liquid can be expressed as follows:

$$
Q_{\max }=\frac{\omega_{0} L_{\mathrm{Q} 1}}{R_{\mathrm{Q} 3}} .
$$

Substituting $R_{\mathrm{Q} 3}$ and $L_{\mathrm{Q} 1}$ into (7), the maximum quality factor of the resonator can be expressed as the following relation of the frequency, liquid density, and liquid viscosity:

$$
\begin{gathered}
Q_{\max } \approx \frac{1}{\sqrt{f_{0}}} \sqrt{\frac{\bar{c}_{66} \rho_{q} \pi}{4}} \frac{1}{\sqrt{\rho \eta}}, \\
Q_{\max } \cdot \sqrt{f_{0}} \cdot \sqrt{\rho \eta} \approx 7.8 \times 10^{6}\left(\mathrm{~kg} \cdot \mathrm{m}^{-2} \cdot \mathrm{s}^{-1}\right),
\end{gathered}
$$




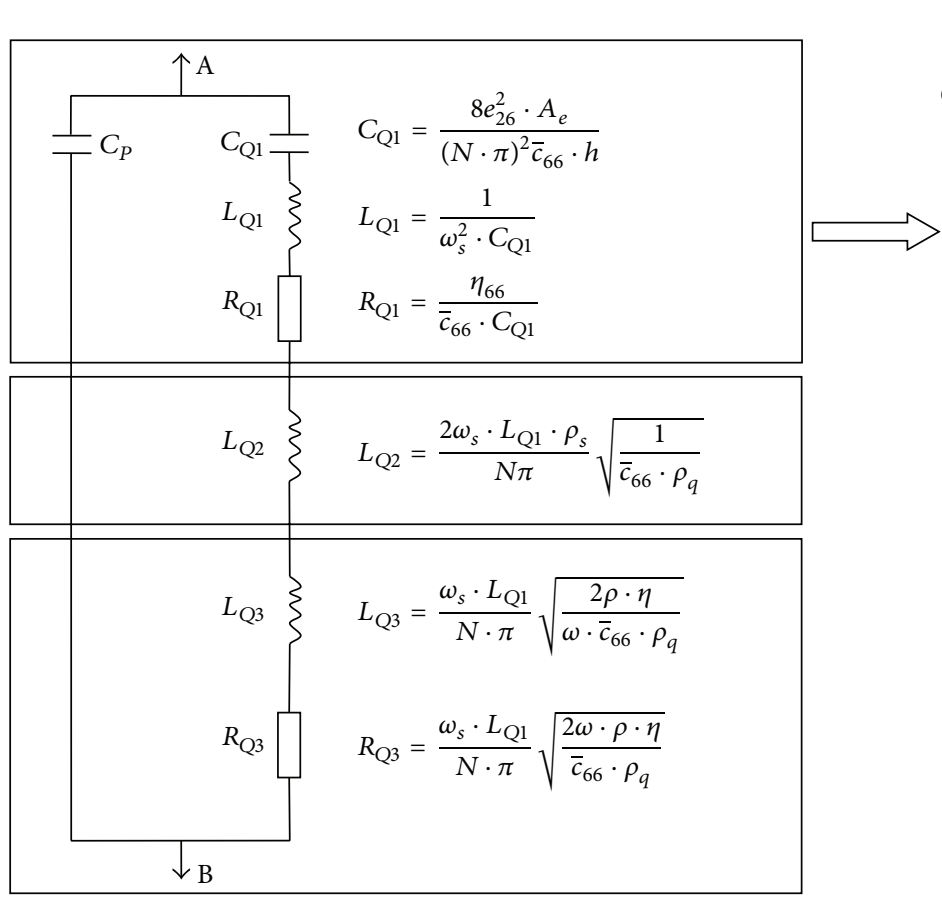

(a)

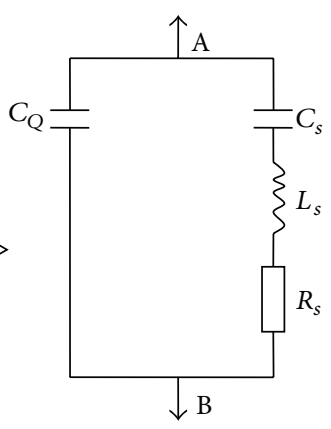

$f=\frac{1}{2 \pi \sqrt{L_{s} \cdot C_{s}}}$

$\mathrm{Q}=\frac{\omega L_{s}}{R_{s}}$

FIGURE 3: Circuit of the sensor: (a) actual model; (b) simplified model.

where $\bar{c}_{66}$ denotes shear stiffness constant of the quartz crystal, $\rho$ is the quartz density, and $f_{0}$ is the nominal oscillation frequency.

Thus, the smallest Allan variance increases with the frequency, liquid density, and liquid viscosity, and it can be expressed as follows:

$$
\sigma_{y}(\tau)_{\min }=1.28 \times 10^{-14} \sqrt{f_{0}} \sqrt{\rho \eta} .
$$

Then, the minimum limit of oscillator frequency is calculated as follows:

$$
\Delta f_{\min }=1.28 \times 10^{-14} f_{0}^{3 / 2} \sqrt{\rho \eta} .
$$

According to (5), the resolution of the FPS sensor is computed as follows:

$$
R=2 \times 10^{-8} \cdot \sqrt{\rho \eta} .
$$

Despite the fact that the noise characterized by $\sigma_{y}$ rises as the oscillator frequency is increased, the resolution has no relation to the nominal frequency of the quartz crystal and liquid density. In addition, the resolution becomes greater when the product of the liquid density and viscosity increases, which means that the resolution is sensitive to the viscosity and density of the liquid.

\section{Temperature Compensation of the FPS Sensor}

Temperature compensation can improve the accuracy and reliability of the sensor [28]. Most of the contaminants in lubricant oil, such as the moisture, gas impurities, and electrolyte impurities, have influences on its dielectric constant. Dielectric constant can be considered as a comprehensive index of the contaminant level, and it can be utilized to indirectly indicate the contaminant degree of lubrication oil. In this paper, we determined the contaminant level through measuring the dielectric constant. When the dielectric constant is large, the purifier will continue to operate until its value reaches a standard level. FPS2800B12C4 fluid property sensor has many advantages, such as small volume, high sensitivity, high reliability, and good dynamic response. However, its dielectric constant is sensitive to temperature change. The changes of distance and area between two electrodes in the sensor caused by temperature change generate output signal errors of dielectric constant. There exists a negative correlation between dielectric constant and temperature. The variation value of dielectric constant, $\varepsilon(\Delta T)$, is a function of the temperature deviation between maximum and minimum values, $\Delta T$. This relation can be expressed by quadratic exponential function or first-order inverse. The first-order inverse function has several advantages over quadratic exponential function, such as easy calculation and small fitting error. Thus, the variation value of dielectric constant is defined as the following first-order inverse function:

$$
\varepsilon(\Delta T)=\frac{a_{1} \cdot \Delta T+b_{1}}{a_{2} \cdot \Delta T+b_{2}}
$$

where $a_{i}$ and $b_{i}(i=1,2)$ are temperature compensation coefficients and $\Delta T=T_{\max }-T_{\min }$ denotes the temperature deviation between maximum and minimum values. 


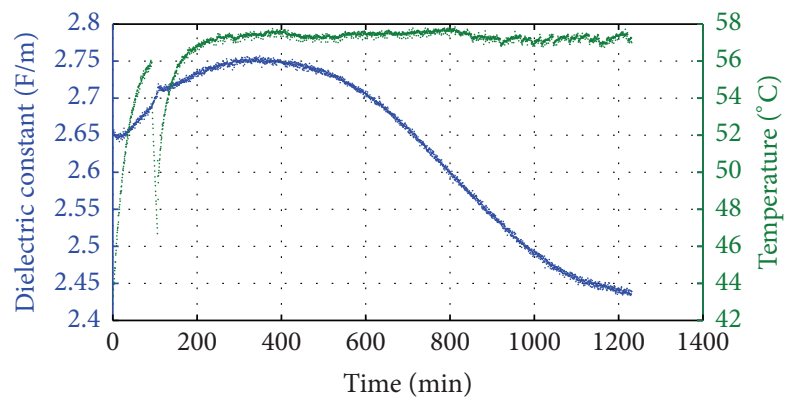

Dielectric constant

Temperature

FIGURE 4: Fitting curves of original data of dielectric constant and temperature.

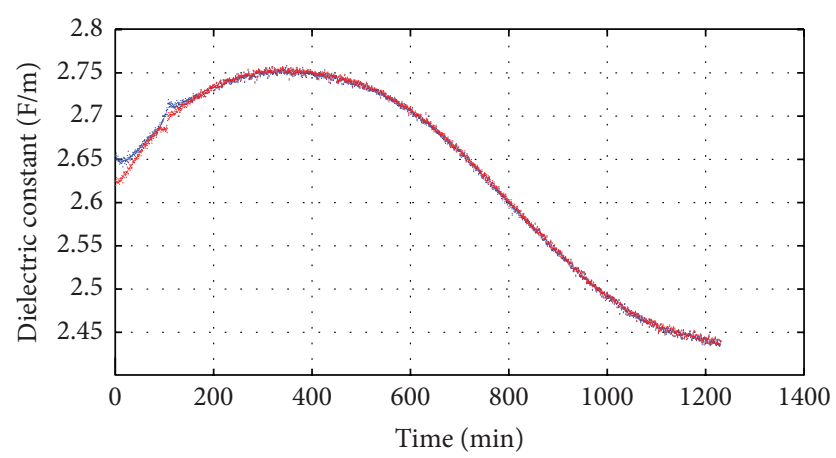

Dielectric constant without compensation

Dielectric constant with compensation

FIGURE 5: Fitting curves of dielectric constant with and without compensation.

As a result, the real dielectric constant can be calculated as follows:

$$
\varepsilon=\varepsilon_{\mathrm{FPS}}-\varepsilon(\Delta T)+\varepsilon_{0},
$$

where $\varepsilon_{\mathrm{FPS}}$ is the dielectric constant value measured by the sensor in real time, $\varepsilon(\Delta T)$ is the variation value of dielectric constant caused by temperature, and $\varepsilon_{0}$ is the dielectric constant value under standard temperature.

\section{Numerical Simulation and Discussion}

During the purification of oil, the purifier generates a large amount of heat. The temperature rises rapidly from room temperature in the first stage, and then it fluctuates in a certain range due to forced or natural cooling. Eventually it converges to a certain value. The stable operating temperature of the sensor is maintained at $57 \sim 58^{\circ} \mathrm{C}$. Thus, the standard temperature $\left(T_{0}\right)$ of the sensor is set to $57^{\circ} \mathrm{C}$, and the dielectric constant corresponding to the temperature is defined as the standard dielectric constant $\left(\varepsilon_{0}\right)$ of the sensor. Oil and water mixed at the ratio of $10: 1$ are to be purified, and purification time is set to 20 hours. In the experiment, the FPS sensor sends a set of experimental data at an interval of 30 seconds to its host controller. The original datum of

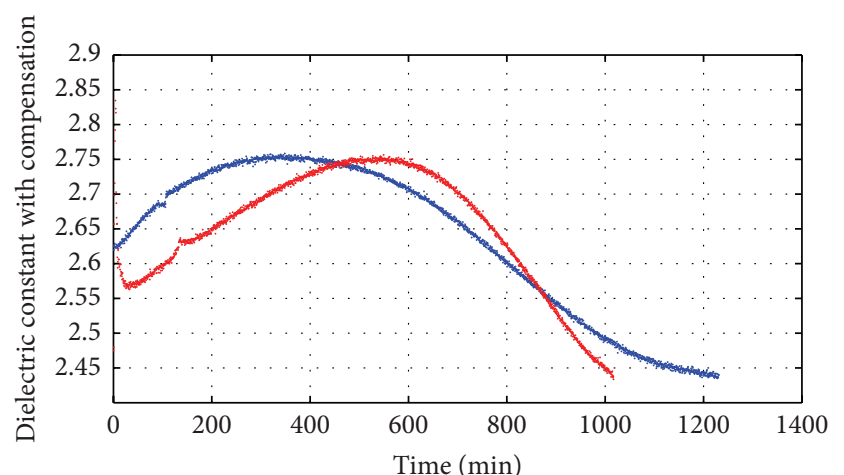

Without microwave

With microwave

FIgURE 6: Purification efficiency comparison with microwave and without microwave.

dielectric constant and temperature is shown in Figure 4. Based on these original data, we can calculate the values of the compensation coefficients in (12) by the fitting method. The calculation results are illustrated as follows: $a_{1}=2.166$, $b_{1}=197.2, a_{2}=1$, and $b_{2}=74.99$. Under standard temperature $T_{0}$, the dielectric constant value $\varepsilon_{0}$ is equal to 2.43. Then, the real dielectric constant after compensation can be calculated according to (13). The dielectric constants after compensation, $\varepsilon$, and without compensation, $\varepsilon_{\mathrm{FPS}}$, are illustrated in Figure 5.

As shown in Figure 4, the temperature varies strongly in the first 200 minutes, then it remains stable, and eventually it converges to $57^{\circ} \mathrm{C}$. Figure 5 demonstrates that high temperature results in big dielectric constant value, and dielectric constant value with compensation is smaller than that without compensation. After 20 hours, the dielectric constant generally tends to be a stable value, which indicates the complete purification of water from oil. Thus, the dielectric constant at this time is defined as the standard dielectric constant under standard temperature.

\section{Method to Improve Purification Efficiency}

As shown in Figure 5, the time for complete purification of water from the lubrication oil is about 20 hours, which means that the vacuum centrifugal solid-liquid-gas purifier is an equipment of low purification efficiency. Microwave is a type of radio wave with a limited frequency band of $0.3 \mathrm{GHz} 300 \mathrm{GHz}$, and it is able to penetrate the ionosphere [29]. Under the nonthermal effects of the microwave field, water molecules tend to be polarized, and their rotation frequency is in the range of microwave frequency. In this way, the original large water droplets become small and might even turn into a single water molecule. Thus, water volatilization is accelerated; the purification speed can also be increased. Figure 6 illustrates the comparison of purification efficiency with and without microwave. It can be noted that, with microwave method, the purification time is about three hours shorter than that without microwave. Therefore, 
microwave method improves the purification efficiency and reduces the purification time.

\section{Conclusion}

A fluid property sensor (FPS) based on tuning-fork technology is utilized to measure the contaminant level of lubricant oil. Density, dielectric constant, viscosity, and temperature can simultaneously be measured by this sensor. The performance characteristics, such as sensitivity coefficient, resolution, and quality factor of the FPS sensor, are analyzed. The resolution is also derived to verify the measurement principle of FPS sensor. Numerical simulation results demonstrate that the resolution of the sensor is only related to the density and viscosity of the lubrication oil.

A temperature compensation method is proposed to eliminate the effect of temperature on measuring parameters, and its validity is investigated by numerical simulations of sensitivity, oscillator frequency, and dielectric constant. Experimental comparisons of purification efficiency using microwave and without microwave are performed. The results show that microwave can improve the purification efficiency.

\section{Conflict of Interests}

The authors declare that there is no conflict of interests regarding the publication of this paper.

\section{Acknowledgments}

This work was supported by the National Natural Science Foundation of China with Grant no. 51375264 and State Key Laboratory of Robotics and System (HIT) with Grant no. SKLRS-2015-MS-06. This work was also partially supported by China Postdoctoral Science Foundation with Grant nos. 2014T70632 and 2013M530318 and Research Awards Fund for Excellent Young and Middle-Aged Scientists of Shandong Province with Grant no. BS2013ZZ008.

\section{References}

[1] A. Chouchene, M. Jeguirim, and G. Trouve, "Biosorption performance, combustion behavior, and leaching characteristics of olive solid waste during the removal of copper and nickel from aqueous solutions," Clean Technologies and Environmental Policy, vol. 16, no. 5, pp. 979-986, 2014.

[2] N. Rotich, R. Tuunila, and M. Louhi-Kultanen, "Modeling and simulation of gravitational solid-solid separation for optimum performance," Powder Technology, vol. 239, pp. 337-347, 2013.

[3] K. P. Galvin and J. E. Dickinson, "Particle transport and separation in inclined channels subject to centrifugal forces," Chemical Engineering Science, vol. 87, pp. 294-305, 2013.

[4] F. Suñol and R. González-Cinca, "Effects of momentum flux and separation distance on bubbly jet impingement in microgravity conditions," Chemical Engineering Science, vol. 97, no. 28, pp. 272-281, 2013.

[5] P. G. Talavera, "Selecting gas/liquid purifiers," Hydrocarbon Process, vol. 69, pp. 81-90, 1990.
[6] A. Krasinski, "A numerical model of droplets coalescence and drainage in fibrous structures," Chemical Engineering Transactions, vol. 32, pp. 1495-1500, 2013.

[7] Y. H. Zhou, W. B. Wu, and K. Q. Qiu, "Recovery of materials from waste printed circuit boards by vacuum pyrolysis and vacuum centrifugal separation," Waste Management, vol. 30, no. 11, pp. 2299-2304, 2010.

[8] D. Zirkler, F. Lang, and M. Kaupenjohann, “'Lost in filtration'the separation of soil colloids from larger particles," Colloids and Surfaces A: Physicochemical and Engineering Aspects, vol. 399, pp. 35-40, 2012.

[9] V. Batalović, "Centrifugal separator, the new technical solution, application in mineral processing," International Journal of Mineral Processing, vol. 100, no. 3-4, pp. 86-95, 2011.

[10] H. Ichiura, T. Nakatani, and Y. Ohtani, "Separation of pulp and inorganic materials from paper sludge using ionic liquid and centrifugation," Chemical Engineering Journal, vol. 173, no. 1, pp. 129-134, 2011.

[11] Y. Chen, G. Y. Ma, S. R. Ning, Y. L. Hu, and J. Gao, "Numerical investigation of bubble motion behavior in a centrifugal vacuum gas-liquid-solid separator for the treatment of contaminated hydraulic fluid," Separation Science and Technology, vol. 50, no. 6, pp. 865-873, 2015.

[12] R. Tambun, K. Nakano, M. Shimadzu, Y. Ohira, and E. Obata, "Sizes influences of weighing bar and vessel in the buoyancy weighing-bar method on floating particle size distribution measurements," Advanced Powder Technology, vol. 23, no. 6, pp. 855-860, 2012.

[13] T. Barth, G. Lecrivain, and U. Hampel, "Particle deposition study in a horizontal turbulent duct flow using optical microscopy and particle size spectrometry," Journal of Aerosol Science, vol. 60, pp. 47-54, 2013.

[14] J. Wang, L. Zhang, F. Lu, and X. Wang, "The segmentation of wear particles in ferrograph images based on an improved ant colony algorithm," Wear, vol. 311, no. 1-2, pp. 123-129, 2014.

[15] C. Guo, W. Liang, and L. Zhang, "The semi-quantitative evaluation method and application of the risks of geological disaster of the Shaan-Jing pipeline," Computer Modelling and New Technologies, vol. 18, no. 4, pp. 126-130, 2014.

[16] P. Kiełczyński, M. Szalewski, A. Balcerzak et al., "Determination of physicochemical properties of diacylglycerol oil at high pressure by means of ultrasonic methods," Ultrasonics, vol. 54, no. 8, pp. 2134-2140, 2014.

[17] G. Arnold, S. Schuldt, Y. Schneider et al., "The impact of lecithin on rheology, sedimentation and particle interactions in oilbased dispersions," Colloids and Surfaces A: Physicochemical and Engineering Aspects, vol. 418, pp. 147-156, 2013.

[18] J. M. Castillo-Secilla, J. Manuel Palomares, and J. Olivares, "Temperature-compensated clock skew adjustment," Sensors, vol. 13, no. 8, pp. 10981-11006, 2013.

[19] Z.-F. Wang, J. Wang, Q.-M. Sui et al., "Development and application of smart geogrid embedded with fiber Bragg grating sensors," Journal of Sensors, vol. 2015, Article ID 108209, 10 pages, 2015.

[20] R. Song, X. Chen, C. Shen, and H. Zhang, "Modeling FOG drift using back-propagation neural network optimized by artificial fish swarm algorithm," Journal of Sensors, vol. 2014, Article ID 273043, 6 pages, 2014.

[21] A. Cao-Paz, L. Rodriguez-Pardo, and J. Fariña, “Temperature compensation of QCM sensors in liquid media," Sensors and Actuators B: Chemical, vol. 193, pp. 78-81, 2014. 
[22] S. Chakraborty, B. Kramer, and B. Kroposki, "A review of power electronics interfaces for distributed energy systems towards achieving low-cost modular design," Renewable and Sustainable Energy Reviews, vol. 13, no. 9, pp. 2323-2335, 2009.

[23] D. Johannsmann and G. Brenner, "Frequency shifts of a quartz crystal microbalance calculated with the frequency-domain lattice-Boltzmann method: application to coupled liquid mass," Analytical Chemistry, vol. 87, no. 14, pp. 7476-7484, 2015.

[24] D. Linden and T. B. Reddy, Handbook of Batteries, McGraw-Hill, New York, NY, USA, 2002.

[25] H. Itoh and Y. Yamada, "Measurement of silicone rubber using impedance change of a quartz-crystal tuning-fork tactile sensor," Japanese Journal of Applied Physics, Part 1: Regular Papers and Short Notes and Review Papers, vol. 45, no. 5, pp. 46434646, 2006.

[26] R. Hernández-Pérez, "Allan deviation analysis of financial return series," Physica A: Statistical Mechanics and its Applications, vol. 391, no. 9, pp. 2883-2888, 2012.

[27] J. Zhai, L. Dong, S. Zhang, F. Lu, and L. Hu, "Different variances used for analyzing the noise type of atomic clock," Applied Mechanics and Materials, vol. 229-231, pp. 1980-1983, 2012.

[28] V. Matko and M. Milanović, "Temperature-compensated capacitance-frequency converter with high resolution," Sensors and Actuators A: Physical, vol. 220, pp. 262-269, 2014.

[29] M. Athar, U. Farooq, S. Z. Ali, and M. Salman, "Insight into the binding of copper(II) by non-toxic biodegradable material (Oryza sativa): effect of modification and interfering ions," Clean Technologies and Environmental Policy, vol. 16, no. 3, pp. 579-590, 2014. 


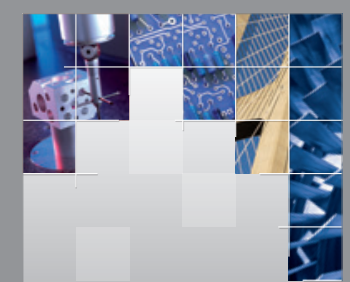

\section{Enfincering}
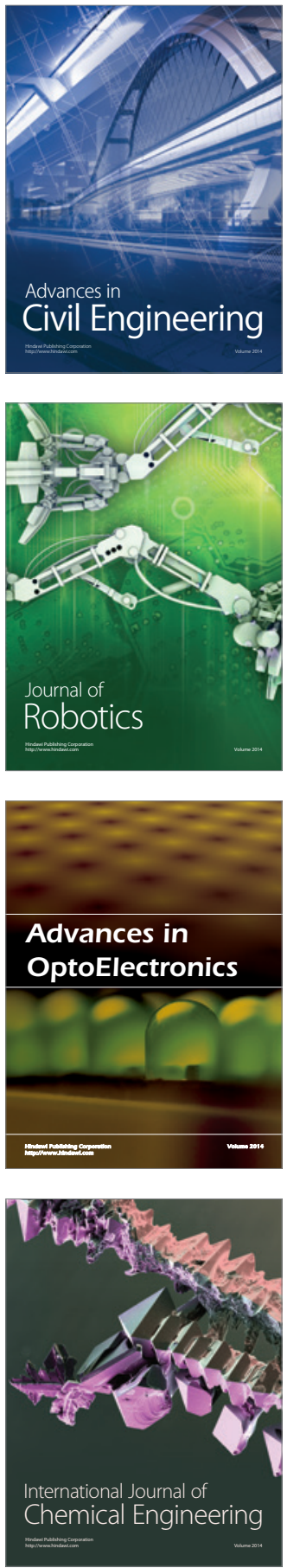

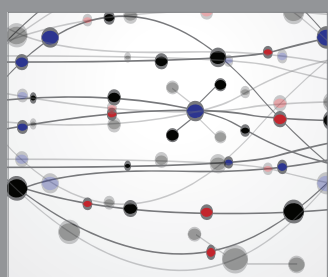

The Scientific World Journal

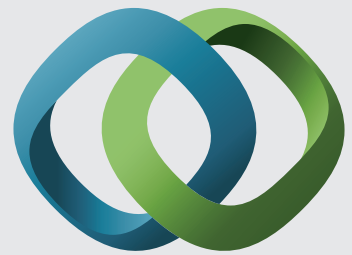

\section{Hindawi}

Submit your manuscripts at

http://www.hindawi.com
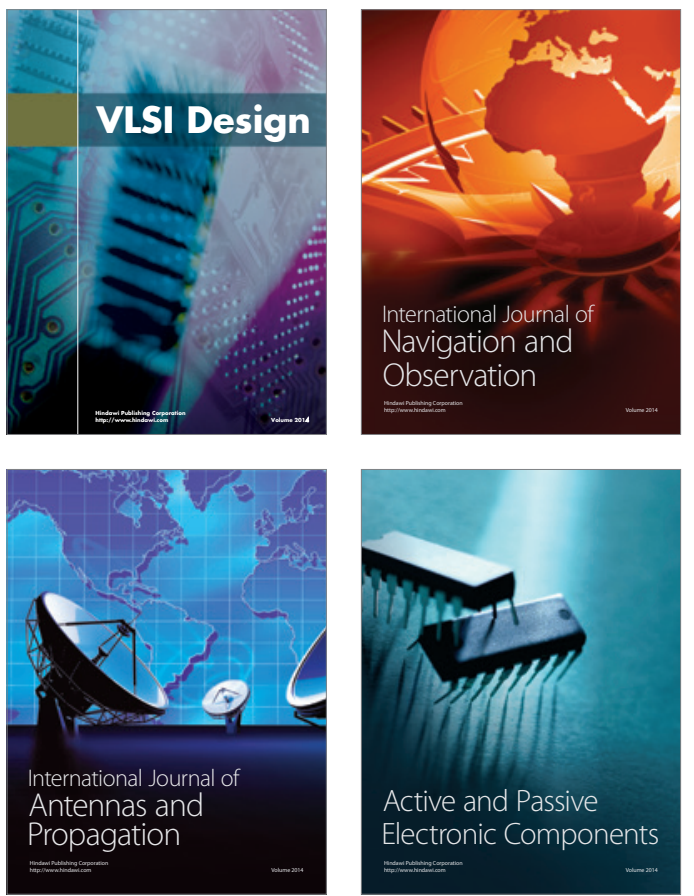
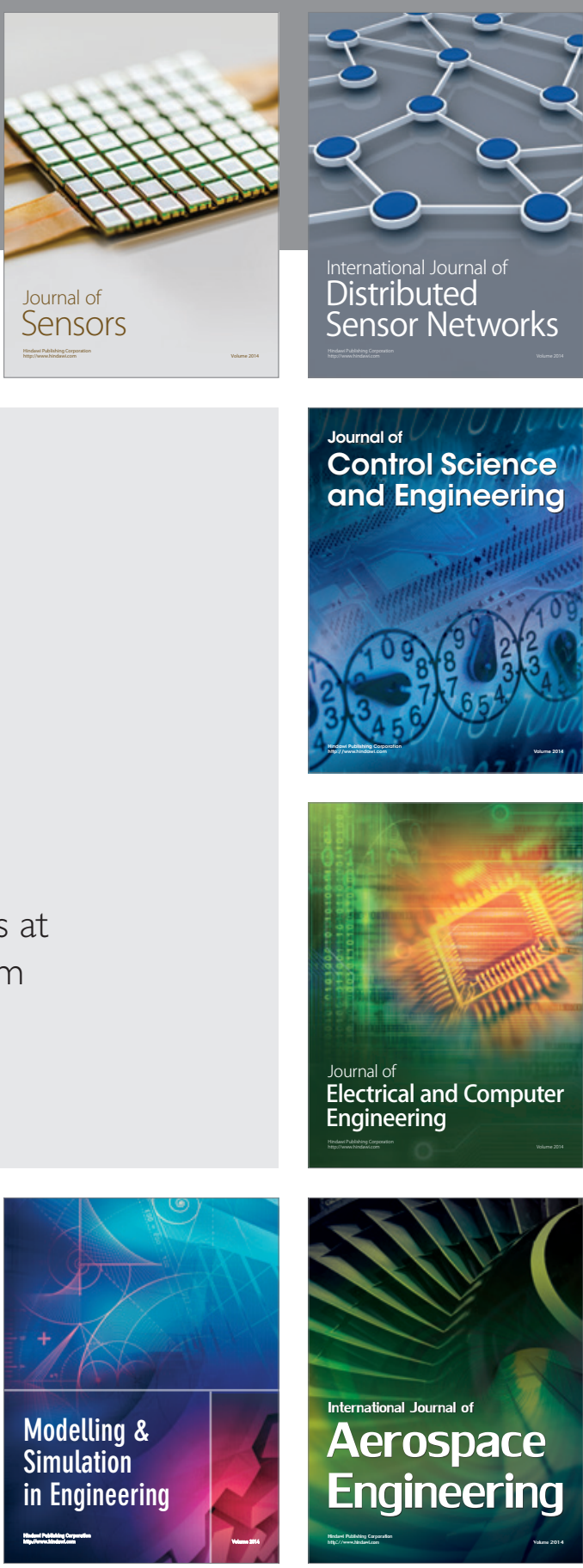

International Journal of

Distributed

Sensor Networks

Journal of

Control Science

and Engineering
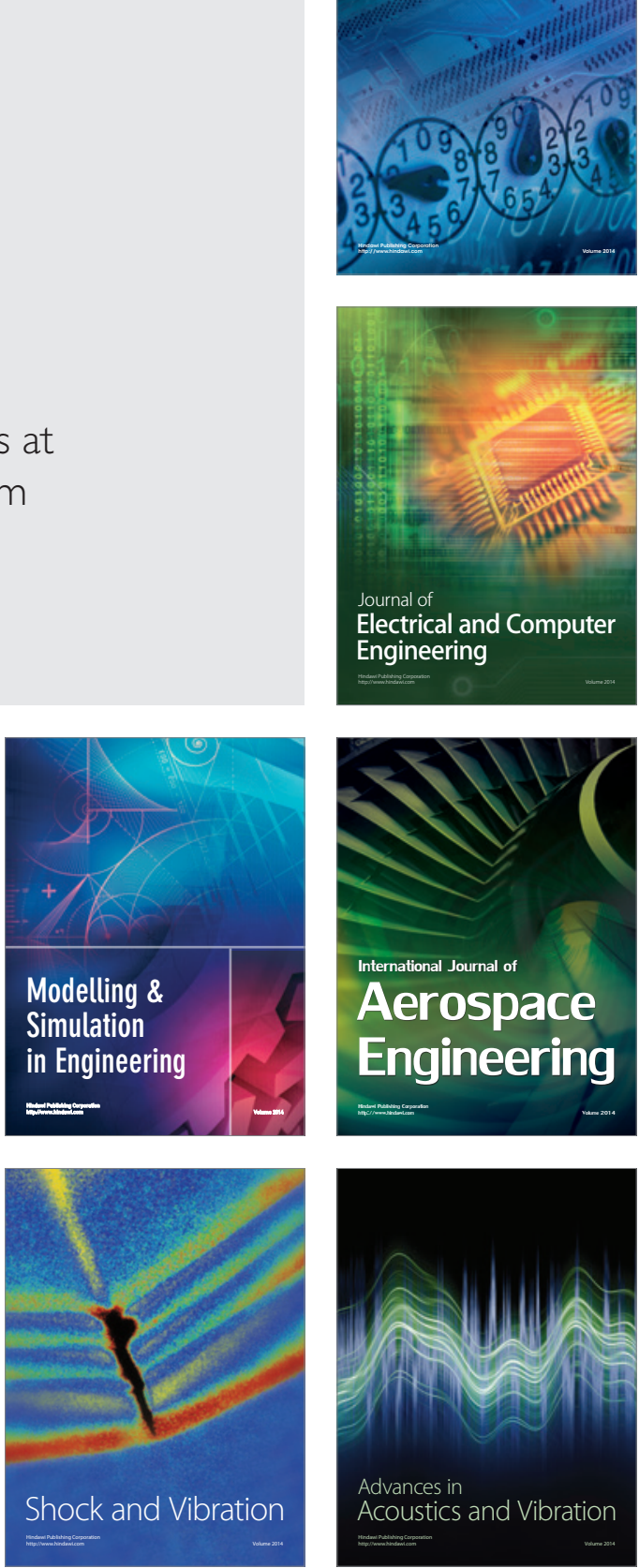